\title{
Grenzen der Integration - wie die Intensivierung der Wirtschaftsintegration zur Gefahr für die politische Integration wird
}

\author{
Martin Höpner und Armin Schäfer*
}

Seit einigen Jahren wird diskutiert, ob und unter welchen Umständen die Europäische Union demokratisiert werden kann. Pessimisten bestreiten die Demokratiefähigkeit der Europäischen Union, weil es keine belastbare kollektive europäische Identität gibt, die sich auf eine geteilte Kommunikations-, Erfahrungs- und Erinnerungsgemeinschaft stützen könnte. ${ }^{1}$ Ihnen entgegnen Optimisten, dass auch nationale Identitäten kein vorgefundenes Substrat abbilden, sondern historisch gewachsen und erst Ergebnis eines demokratischen Prozesses sind. Prinzipiell spricht aus dieser Sicht nichts dagegen, dass eine europäische Identität an die Seite nationaler Identitäten tritt. ${ }^{2}$ Erreicht werden kann dies am ehesten durch eine Politisierung der Gemeinschaft, denn nur dann werden sich die nationalen Öffentlichkeiten füreinander öffnen. Europäisierte nationale Öffentlichkeiten bilden ihrerseits die Bedingung für eine über den Nationalstaat hinausreichende Solidarität, die normativ anspruchsvolle Politiken auf europäischer Ebene möglich macht. ${ }^{3}$

Auf den ersten Blick scheinen die Voraussetzungen zur Entstehung einer kollektiven europäischen Identität heute günstiger als in der Vergangenheit. Sowohl der Vertrag über eine Verfassung für Europa als auch einige Richtlinien sowie Urteile des Europäischen Gerichtshofs (EuGH) haben in den letzten Jahren ein erhebliches Maß an Aufmerksamkeit erfahren. Im Vergleich zur Phase vor ,Maastricht' hat eine Politisierung europäischer Politik stattgefunden. ${ }^{4}$ Allerdings verlaufen Konflikte häufig entlang nationaler Grenzen. Gegen die von supranationalen Akteuren forcierte Marktöffnung lassen sich nationalstaatliche Abwehrreaktionen beobachten, die europäischer Solidarität zuwiderlaufen: In Großbritannien streiken Gewerkschaften unter dem Slogan „,britische Jobs für britische Arbeiter“ gegen portugiesische und italienische Bauarbeiter; ${ }^{5}$ im Zuge der Finanzkrise drängt der französische Präsident, Arbeitsplätze der Automobilindustrie aus Mittel- und Osteuropa nach Frankreich zu verlagern; ${ }^{6}$ und Deutschland verlängert die Beschränkungen der Freizügigkeit für Arbeitskräfte aus den Beitrittsstaaten bis 2011. ${ }^{7}$ Statt einer Politisierung, die eine kollektive Identi-

* PD Dr. Martin Höpner, Forschungsgruppenleiter, Max-Planck-Institut für Gesellschaftsforschung, Köln. Dr. Armin Schäfer, Wissenschaftlicher Mitarbeiter, Max-Planck-Institut für Gesellschaftsforschung, Köln.

1 Dieter Grimm: Braucht Europa eine Verfassung?, in: Marie-Theres Tennefeld/Lothar Philipps/Susanne Heil (Hrsg.): Informationsgesellschaft und Rechtskultur in Europa, Baden-Baden 1995, S. 211-230; Peter Graf Kielmansegg: Lässt sich die Europäische Union demokratisch verfassen?, in: Frank Decker/Marcus Höreth (Hrsg.): Die Verfassung Europas. Perspektiven des Integrationsprojektes, Wiesbaden 2009, S. 219-236.

2 Jürgen Habermas: Eine Diskussionsbemerkung zu Dieter Grimm: Braucht Europa eine Verfassung?, in: MarieTheres Tennefeld/Lothar Philipps/Susanne Heil (Hrsg.): Informationsgesellschaft und Rechtskultur in Europa, Baden-Baden 1995, S. 231-235.

3 Jürgen Habermas: Europapolitik in der Sackgasse. Plädoyer für eine Politik der abgestuften Integration, in: Jürgen Habermas: Ach, Europa, Frankfurt 2008, S. 96-127, hier S. 106-107.

4 Liesbet Hooghe/Gary Marks: A Postfunctionalist Theory of European Integration: From Permissive Consensus to Constraining Dissensus, in: British Journal of Political Science 1/2009, S. 1-23.

5 The Economist: Discontents, wintry and otherwise, 07.02.2009, S. 31.

6 Süddeutsche Zeitung: Sarkozy verärgert Kanzlerin Merkel, Bundesregierung nennt Pariser Hilfsprogramm für Autoindustrie „Protektionismus“, 12.02.2009; Süddeutsche Zeitung: Sorge um deutsche Exporte, 12.02.2009.

7 Frankfurter Allgemeine Zeitung: Keine Freizügigkeit für Osteuropäer bis 2011, 29.04.2009. 
tät fördert, droht eine ,,negative Politisierung“, 8 die Unterschiede zwischen den Mitgliedstaaten oder sozialen Gruppen verschärft.

Die Auseinandersetzungen der vergangenen Jahre waren vielfach das Resultat von Verteilungskonflikten zwischen den Mitgliedstaaten. So verliefen die Konfliktlinien bei der Dienstleistungsrichtlinie ${ }^{9}$ und der Übernahmerichtlinie ${ }^{10}$ vor allem zwischen Mitgliedstaaten mit unterschiedlichen Kapitalismus- und Wohlfahrtsstaatsmodellen, weniger hingegen entlang der Links-rechts-Achse der Parteiensysteme. Ähnliches gilt, wie im Einzelnen zu zeigen sein wird, für einige richtungsweisende Urteile des EuGH. Zudem hat die Distanz zwischen europäischen Eliten und Teilen der Bevölkerung in den letzten beiden Jahrzehnten zugenommen. Insbesondere diejenigen, die von einer verstärkten Konkurrenz aus den Beitrittsländern negativ betroffen sind, entwickeln integrationsskeptische Einstellungen. Der permissive Konsens der Vergangenheit wird durch ein generalisiertes Misstrauen gegenüber den Eliten abgelöst. ${ }^{11}$ Diese Trends stehen in auffälligem Kontrast zur neuesten Rechtsprechung des EuGH zur Unionsbürgerschaft, die Individualrechte ausweitet und zunehmend (finanzielle) Solidarität zwischen Angehörigen unterschiedlicher Mitgliedstaaten einfordert. Der Gerichtshof unterstellt in seinen Urteilen, dass bereits eine ,,belastbare Identität der Europäer als Europäer"12 existiert.

Wir nehmen diese aktuellen Entwicklungen zum Anlass einer Analyse des Stands der europäischen Integration, wobei ein spezifischer Aspekt im Zentrum der Betrachtung steht: die Wirtschafts- und Sozialintegration. Dabei ist einzuräumen, dass zahlreiche Gesichtspunkte außer Acht bleiben, von der Gemeinsamen Außen- und Sicherheitspolitik über die Fortschritte bei der Koordination der mitgliedstaatlichen Bildungssysteme bis hin zur Klimapolitik. Zeigen lässt sich jedoch, dass sich wesentliche, wenn auch gewiss nicht alle Dynamiken und Eigenarten der gegenwärtigen Integrationsphase durch Einnahme einer an die ,ökonomische Basis “ rückgekoppelten Perspektive verstehen lassen. Speziell gilt es zu verdeutlichen, dass die höchst erfolgreiche Marktintegration eine Tiefe erreicht hat, die Gefahr läuft, die Schaffung eines transnationalen , politischen Raums ' und damit die Entstehung eines politischen Gemeinwesens zu erschweren. Die zur weiteren marktvertiefenden Integration bereitliegenden Instrumente sollten daher behutsam genutzt werden, um innereuropäische Kooperation und die Entwicklung gemeinsamer Problemperzeptionen zu erleichtern.

Das Argument wird in vier Schritten entwickelt. Zunächst erfolgt eine Bestandsaufnahme der gestiegenen Heterogenität der Europäischen Union. Darauf aufbauend wird diskutiert, wie supranationale Institutionen Handlungsspielräume gewinnen, die Integration auch gegen den Widerstand potenziell integrationsunwilliger Mitgliedstaaten weiterentwickeln zu können. Im nächsten Schritt wird gefragt, wie sich die Kombination von gestiegener Heterogenität und sich verändernden Handlungsspielräumen auf die Wirtschaftsintegration ${ }^{13}$ auswirkt, wobei drei Dimensionen unterschieden werden: marktgestaltende Integration, marktschaffende Integration sowie die Schaffung eines europäischen Antidiskriminierungs-

8 Diesen Begriff verwendet Claus Offe: Demokratie und Wohlfahrtsstaat: Eine europäische Regimeform unter dem Stress der europäischen Integration, in: Wolfgang Streeck (Hrsg.): Internationale Wirtschaft, nationale Demokratie. Herausforderungen für die Demokratietheorie, Frankfurt 1998, S. 99-136, hier S. 113.

9 Richtlinie 2006/123/EG des Europäischen Parlaments und des Rates vom 12. Dezember 2006 über Dienstleistungen im Binnenmarkt, in: Amtsblatt der EU, Nr. L 376 vom 27. Dezember 2006, S. 36-68.

10 Richtlinie 2004/25/EG des Europäischen Parlaments und des Rates vom 21. April 2004 betreffend Übernahmeangebote, in: Amtsblatt der EU, Nr. L 142 vom 30. April 2004, S. 12-23.

11 Eindrücklich Max Haller: Die Europäische Integration als Elitenprozess. Das Ende eines Traums?, Wiesbaden 2009.

12 So formuliert Kielmansegg: Lässt sich die Europäische Union demokratisch verfassen, 2009, S. 228.

13 Wir meinen hiermit stets sowohl marktschaffende als auch marktkorrigierende, also auch sozialpolitische Maßnahmen (,Wirtschafts- und Sozialintegration'). 
raums. Im abschließenden Teil spitzen wir die Ergebnisse der Betrachtung im Hinblick auf ihre Implikationen für die politische Integration zu. Die These lautet, dass angesichts des gegenwärtigen Standes der europäischen Integration, weniger mehr sein “ könnte: Selbstzurückhaltung bei der vor allem mit den Mitteln der Rechtsfortbildung betriebenen Intensivierung der Wirtschaftsintegration könnte mehr politische Integration bewirken als ungebremste ,Integration durch Recht‘.

\section{Gestiegene politökonomische Heterogenität}

Als Ausgangspunkt dient die Beobachtung, dass sich mehrere der jüngeren, hoch politisierten Auseinandersetzungen um europäische Fragen auf ökonomische Interessenkonflikte zurückführen lassen. Die Konflikte um die Dienstleistungsrichtlinie ${ }^{14}$ und um die mit ,Viking ' und ,Laval ' begonnene Serie kontroverser EuGH-Urteile ${ }^{15}$ betreffen die Frage, inwieweit osteuropäische Wettbewerber ihre durch niedrigere Lohnkosten und Arbeitsstandards erzielbaren Wettbewerbsvorteile unter Nutzung des Instruments der Arbeitnehmerentsendung auf den Märkten der mittel- und nordeuropäischen Hochlohnökonomien legitimerweise ausspielen dürfen. Dieser Konflikt ist im Kern ein Konflikt um die Tiefe der Marktintegration, also um den Anwendungsbereich und die Wirksamkeit der europäischen Grundfreiheiten, die seit Beginn der europäischen Integration das Herzstück der europäischen Verträge bilden. ${ }^{16}$ Interessenkonflikte folgen zudem aus der institutionellen Heterogenität europäischer Produktions- und Verteilungsregime, die in einer neueren Terminologie „Spielarten des Kapitalismus“ genannt werden. ${ }^{17}$ Im europäischen Wirtschaftsraum finden sich koordinierte Ökonomien ebenso wie liberale Marktwirtschaften. Solche Unterschiede standen im Zentrum der Auseinandersetzungen um die europäische Übernahmerichtlinie und die auf der Kapitalverkehrsfreiheit beruhenden EuGH-Urteile zu ,Goldenen Aktien '. ${ }^{18}$ Kurz: In der Europäischen Union mit 27 Mitgliedstaaten müssen Interessengegensätze zum Ausgleich gebracht werden, die tiefer gehen als jene der vergleichsweise homogenen EWG6 oder der späteren EG/EU-12 oder -15 .

14 Im Kern drehte sich die Auseinandersetzung um die Frage, inwiefern Arbeits- und Sozialrecht auf zeitweilig entsandte Arbeitnehmer aus anderen Mitgliedstaaten übertragen werden darf. Je enger der Handlungsspielraum der Empfängerländer, umso größer der aus niedrigeren Arbeitsstandards und günstigeren Lohnkosten entstehende Wettbewerbsvorteil der entsendenden Länder. Kritische Beurteilungen der Dienstleistungsrichtlinie waren nach allgemeiner Einschätzung für die Ablehnung des Verfassungsvertrags im französischen Referendum des Jahrs 2005 mitverantwortlich. Siehe Joachim Schild: Ein Sieg der Angst - das gescheiterte französische Verfassungsreferendum, in: integration 3/2005, S. 187-200, hier S. 198; Europäische Kommission: Flash Eurobarometer 171. The European Constitution: Post-referendum survey in France, Luxemburg 2005.

15 EuGH Rs. C-438/05 (Viking), Slg. 2007, I-10779; EuGH Rs. C-341/05 (Laval), Slg. 2007, I-11767. Inhaltliche Hinweise zu den Urteilen folgen in den nachfolgenden Textabschnitten.

16 Die Grundfreiheiten garantieren das Recht auf freie Bewegung auf den europäischen Güter-, Dienstleistungs-, Kapital- und Arbeitsmärkten (Art. 34, 45, 49, 56, 63 Vertrag über die Arbeitsweise der Europäischen Union (AEUV)). Aufgrund der EuGH-Judikatur zur unmittelbaren Wirkung des Gemeinschaftsrechts gelten sie nicht nur zwischen Staaten, sondern etablieren Rechte, auf die sich Wirtschaftsteilnehmer gegenüber den Gebietskörperschaften aller Ebenen berufen können. Vgl. EuGH Rs. C-26/62 (Van Gend en Loos/Administratie der Belastingen), Slg. 1963, 3.

17 Grundlegend Peter A. Hall/David Soskice: An Introduction to Varieties of Capitalism, in: Peter A. Hall/David Soskice (Hrsg.): Varieties of Capitalism. Institutional Foundations of Comparative Advantage, Oxford 2001, S. 1-68.

18 ,Goldene Aktien“ sind Sonderstimmrechte von Gebietskörperschaften auf den Hauptversammlungen von (typischerweise: privatisierten) Unternehmen. 


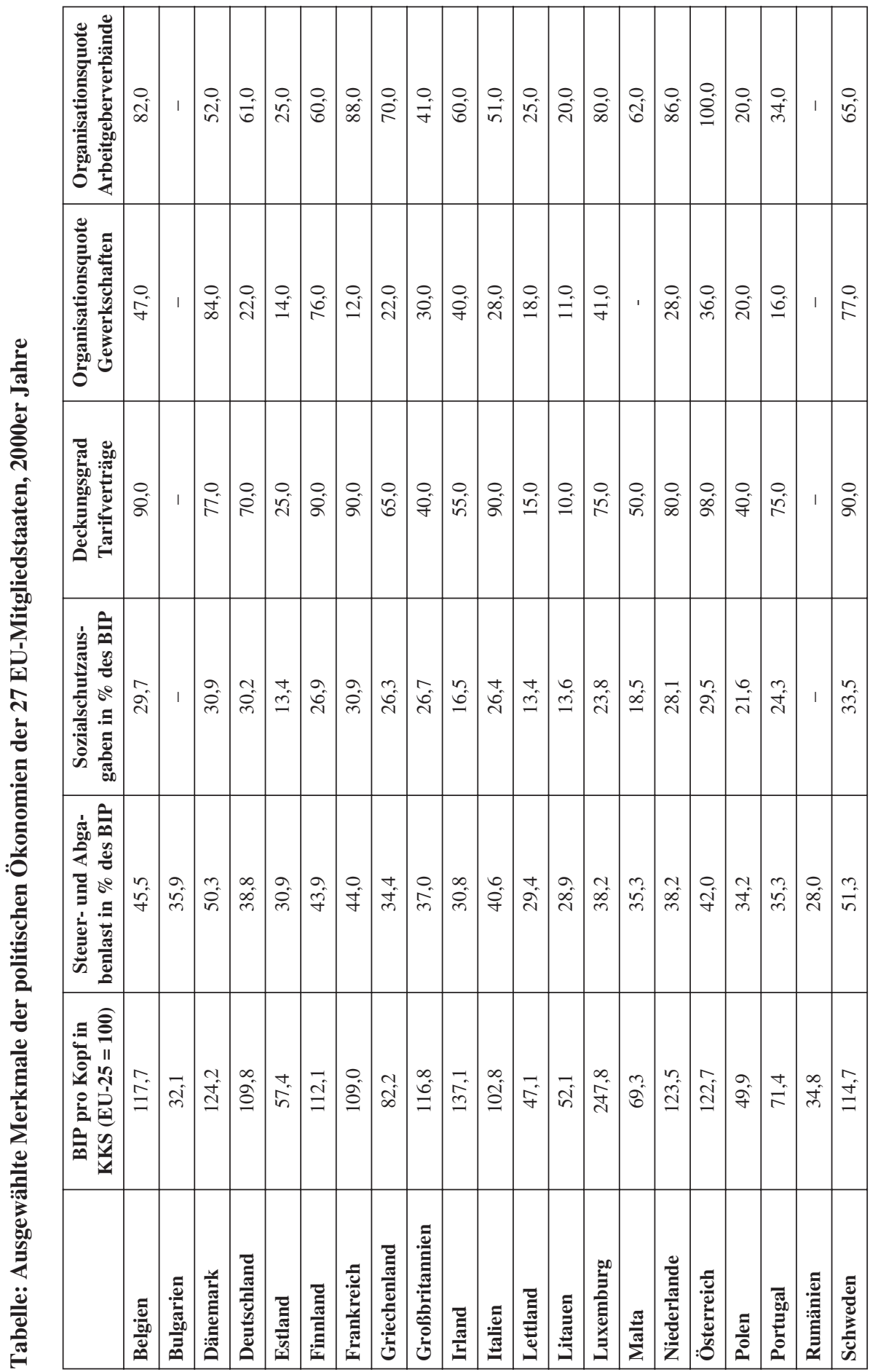




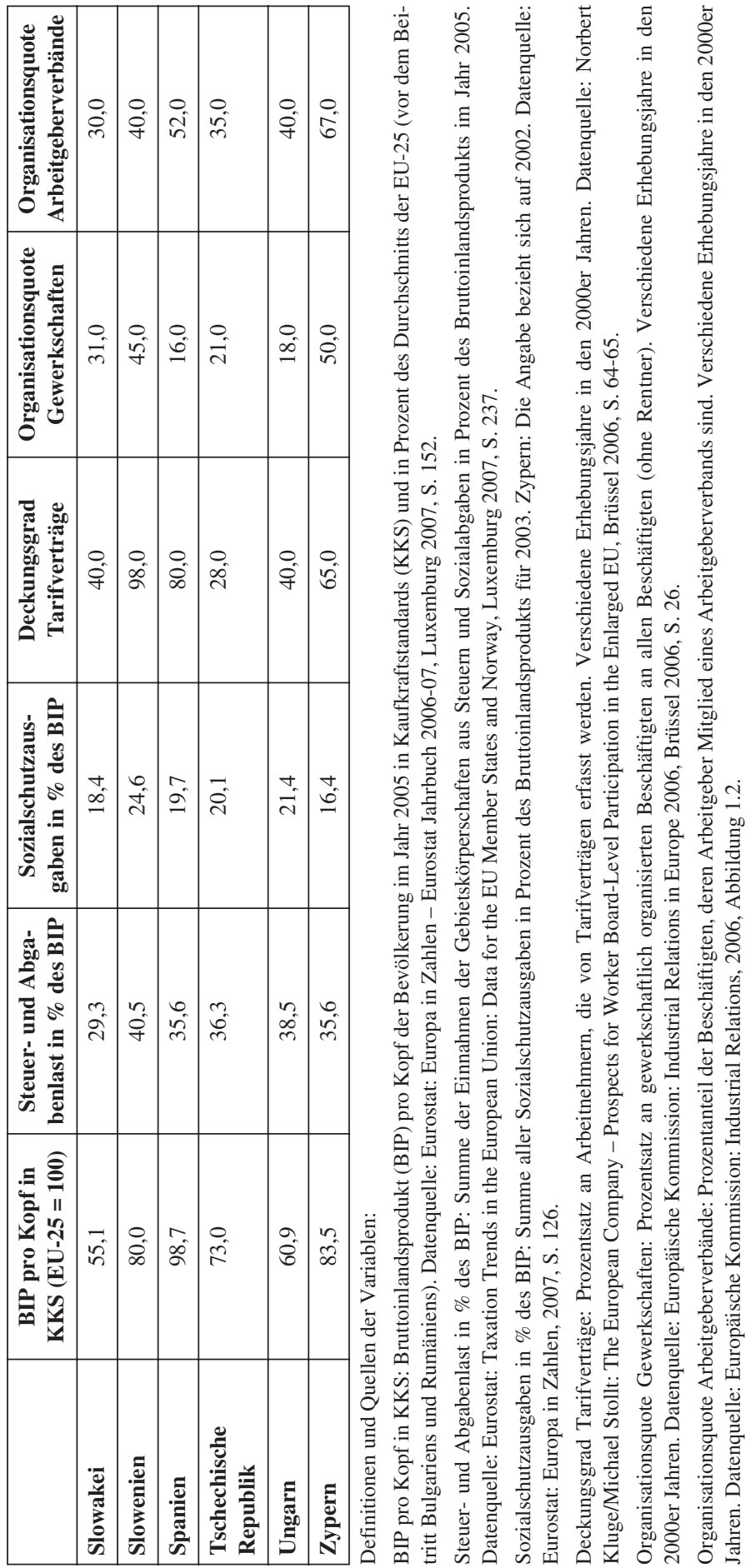


Die EU-27 weist, wie die Daten in der Tabelle illustrieren, ein vorher nicht gekanntes $\mathrm{Maß}$ an Wohlstandsunterschieden auf. Angegeben wird das Pro-Kopf-Bruttoinlandsprodukt der Mitgliedstaaten in Kaufkraftstandards ${ }^{19}$ des Jahres 2005 (vor dem Beitritt Bulgariens und Rumäniens), wobei der Durchschnitt der damaligen EU-25 auf den Wert 100 gesetzt wurde. Diese Wohlstandsunterschiede reflektieren unterschiedliche Produktivitätsniveaus der Volkswirtschaften und schlagen sich in unterschiedlichen Lohnniveaus nieder. Fünf Länder erwirtschaften ein Pro-Kopf-BIP, das mehr als 20 Prozent über dem Durchschnitt liegt: Luxemburg, Dänemark, Irland, die Niederlande und Österreich. Diesen sehr reichen Ländern stehen elf Länder gegenüber, deren Wohlstandsniveau unterhalb von 80 Prozent des Durchschnitts liegt: Portugal, Malta sowie neun der zehn beigetretenen osteuropäischen Transformationsländer (das zehnte dieser Länder, Slowenien, liegt bei genau 80 Prozent des Durchschnitts-BIP). Das 39-Millionen-Einwohnerland Polen erwirtschaftet pro Kopf eine Wertschöpfung, die in etwa der Hälfte des Durchschnitts der EU-25 entspricht.

Nicht minder gravierend sind die institutionellen Unterschiede, die darüber Auskunft geben, wie in den europäischen Spielarten des Kapitalismus Wertschöpfung erwirtschaftet und verteilt wird. Das gilt beispielsweise für die Steuer- und Abgabenquoten. Weniger als 30 Prozent des jährlichen BIP werden in Lettland, Litauen und Rumänien, aber mehr als 50 Prozent in Dänemark und Schweden als Steuern und Abgaben erhoben. ${ }^{20}$ Das korrespondiert mit der heterogenen Konstruktionsweise und Größe europäischer Wohlfahrtsstaaten. Der schlankste und der am stärksten ausgebaute Wohlfahrtsstaat, gemessen in Ausgabenanteilen am nationalen BIP, unterscheiden sich um den Faktor 2,5. ${ }^{21}$ Und auch die Unterschiedlichkeit der institutionellen Voraussetzungen organisierter Sozialpartnerschaft könnte man sich größer kaum vorstellen. So streuen um eine durchschnittliche Netto-Organisationsquote der Gewerkschaften von 33 Prozent der Beschäftigten (ohne Rentner) Litauen mit 11 und Frankreich mit 12 Prozent, aber Schweden mit 77 und Dänemark mit 84 Prozent. Ähnliches gilt, wie die Daten in der Tabelle zeigen, für die Organisationsquoten der Arbeitgeberverbände sowie die Bedeutung von oberhalb der Unternehmensebene ausgehandelten Tarifverträgen. ${ }^{22}$

Vergleichende Politische Ökonomie und Vergleichende Wohlfahrtsstaatsforschung haben unterschiedliche Typologien zur Klassifikation von Produktions- und Verteilungsregimen hervorgebracht. Beispiele sind Hall und Soskices Unterscheidung von koordinierten und liberalen Marktwirtschaften ${ }^{23}$ und Esping-Andersens Unterscheidung von liberalen,

19 Der Kaufkraftstandard (KKS) ist eine statistische Kunstwährung. Er erlaubt aussagekräftige Vergleiche zwischen Wirtschaftsindikatoren, weil bei seiner Errechnung in den Wechselkursen nicht zum Ausdruck kommende Unterschiede zwischen den nationalen Preisniveaus zum Ausgangspunkt genommen werden.

20 Siehe zur Steuerpolitik im internationalen Vergleich Steffen Ganghof: The Politics of Income Taxation. A Comparative Analysis, Colchester 2006; Uwe Wagschal: Steuerpolitik und Steuerreformen im internationalen Vergleich. Eine Ursache der Determinanten, Ursachen und Blockaden, Münster 2005.

21 Siehe zum internationalen Vergleich von Wohlfahrtsstaaten Evelyne Huber/John D. Stephens: Development and Crisis of the Welfare State. Parties and Policies in Global Markets, Chicago 2001; Herbert Obinger/Bernhard Kittel: Parteien, Institutionen und Wohlfahrtsstaat. Politisch-institutionelle Determinanten der Sozialpolitik in OECD-Ländern, in: Herbert Obinger/Uwe Wagschal/Bernhard Kittel (Hrsg.): Politische Ökonomie. Demokratie und wirtschaftliche Leistungsfähigkeit, Wiesbaden 2003, S. 355-384.

22 Siehe zum internationalen Vergleich von Institutionen der Arbeitsbeziehungen Franz Traxler/Sabine Blaschke/ Bernhard Kittel: National Labor Relations in Internationalized Markets. A Comparative Study of Institutions, Change, and Performance, Oxford 2001; Jelle Visser: Union membership statistics in 24 countries, in: Monthly Labor Review 1/2006, S. 38-49.

23 Hall/Soskice: An Introduction to Varieties of Capitalism, 2001. Zudem gehen einige Autoren davon aus, dass sich in den osteuropäischen Beitrittsländern eine eigenständige Spielart des Kapitalismus herausgebildet hat (,Dependent Market Economies'), deren institutionelles Gefüge (so insbesondere auch die Steuersysteme) in besonderem Maß auf Direktinvestitionen internationaler Konzerne zielt. Siehe hierzu Andreas Nölke/Arjan Vliegenthart: Enlarging the Varieties of Capitalism. The Emergence of Dependent Market Economies in East Central Europe, in: World Politics 4/2009, S. 670-702. 
konservativen und sozialdemokratischen Wohlfahrtsstaaten. ${ }^{24}$ Entscheidend ist an dieser Stelle, dass die gesamte Bandbreite von Wohlstands- und Regimeunterschieden der entwickelten Industrieländer sich auch in der Europäischen Union findet. Mit der Zunahme solcher Heterogenitäten sinkt die Wahrscheinlichkeit, dass sich die deutschen oder skandinavischen Modelle von organisiertem Kapitalismus, Sozialpartnerschaft und Wohlfahrtsstaat auf die Europäische Union übertragen lassen. Die für solche Schritte marktgestaltender Integration notwendige Einstimmigkeit erscheint angesichts unterschiedlicher Ausgangslagen und den damit einhergehenden heterogenen Interessen auch auf mittel- bis langfristige Sicht nicht erreichbar. Gleichzeitig aber verändern sich mit zunehmender Heterogenität auch die Interessen an marktschaffender Integration. Sie werden konfrontativer. Denn der von den neuen Mitgliedstaaten präferierten extensiven Interpretation der europäischen Grundfreiheiten und der effektiven transnationalen Öffnung der Dienstleistungsmärkte stehen ebenso deutlich hervortretende Interessen der alten Mitgliedsländer gegenüber, sich vor Niedriglohnkonkurrenz auf heimischen Märkten zu schützen.

\section{Kommission und EuGH als Motoren der Integration}

Angesichts der gestiegenen Interessenheterogenität ist bemerkenswert, dass die Wirtschaftsintegration über unterschiedliche Integrationsdimensionen hinweg keineswegs stagniert. Im Gegenteil, in der jüngeren Vergangenheit lässt sich eine weitere Vertiefung der Wirtschaftsintegration beobachten. Einen bedeutenden Schritt haben die EU-Staaten mit der Gründung der Europäischen Wirtschafts- und Währungsunion unternommen. ${ }^{25}$ Ein erheblicher Teil der gegenwärtigen Vertiefung wirtschaftlicher Integration beruht allerdings nicht auf dem Verhandlungsgeschick nationaler Regierungschefs, sondern auf der Anwendung der Binnenmarktregeln und des Wettbewerbsrechts auf neue Sachverhalte und auf der sukzessiven Weiterentwicklung des europäischen Rechts durch den EuGH. Als ,Motoren der Integration “ erkämpfen sich Kommission und EuGH Spielräume, die es ihnen mal mehr, mal weniger erlauben, Marktintegration auch gegen den Widerstand der Mitgliedstaaten durchzusetzen. $^{26}$

Die Ursache für diese Eigendynamiken der Marktintegration, so eine überzeugende These aus der Integrationsforschung, liegt in der Natur des Europarechts als Ort struktureller Kopplung von Recht und Politik. ${ }^{27}$ Dem europarechtlichen Diskurs und so auch der Rechtsprechung des EuGH liegt ein Primat des ,Telos der Integration', eine Tendenz zur sukzessiven Landnahme gegenüber nationalen Rechtsordnungen zugrunde. So sind die Direktwirkung des Europarechts, dessen Vorrang vor nationalem Recht (einschließlich Verfassungsrecht), die Verpflichtung Privater durch die Grundfreiheiten des Binnenmarkts sowie die Deutung der Grundfreiheiten als Beschränkungsverbote (statt lediglich: Diskriminierungsverbote) nicht das Ergebnis politischer Übereinkünfte, sondern Innovationen der Rechtsprechung des EuGH (vergleiche hierzu den nachfolgenden Abschnitt). ${ }^{28}$ Auch wurde auf die strategische Fähigkeit der Kommission hingewiesen, mit dem EuGH

24 Gøsta Esping-Andersen: The Three Worlds of Welfare Capitalism, Oxford 1990.

25 Siehe hierzu Henrik Enderlein: Nationale Wirtschaftspolitik in der europäischen Währungsunion, Frankfurt/ New York 2005.

26 Grundlegend Mark A. Pollack: The Engines of European Integration. Delegation, Agency, and Agenda Setting in the EU, Oxford 2003, S. 75-154.

27 Grundlegend hierzu Joseph H.H. Weiler: A Quiet Revolution. The European Court of Justice and Its Interlocutors, in: Comparative Political Studies 4/1994, S. 510-534.

28 Siehe hierzu Karen J. Alter/Sophie Meunier-Aitsahalia: Judicial Politics in the European Community. European Integration and the Pathbreaking Cassis de Dijon Decision, in: Comparative Political Studies 4/1994, S. 535-561; Karen J. Alter: Establishing the Supremacy of European Law, Oxford/New York 2001. 
,über Bande' zu spielen und damit mehr Integration zu bewirken, als es auf Grundlage der Präferenzen der Mitgliedsländer zu erwarten wäre. ${ }^{29}$

Haben diese Handlungsspielräume der supranationalen Institutionen in der jüngeren Vergangenheit zu- oder abgenommen? Einige der oben skizzierten Beobachtungen legen nahe, dass die Spielräume enger werden. Dazu zählt die Politisierung europäischer Entscheidungen und damit die geringer werdende Chance, europäische Maßnahmen als quasi-technische Angelegenheiten an Politik und Öffentlichkeit vorbei umzusetzen. Das spektakuläre politische Scheitern der Kommission in zwei Fällen scheint diese Interpretation zu bestätigen. Sowohl im Fall der Übernahmerichtlinie wie auch der Dienstleistungsrichtlinie blieben die erreichten Kompromisse hinter den Vorstellungen der Kommission zurück. ${ }^{30}$ Allerdings wurden beide Entscheidungen durch nachfolgende EuGH-Urteile mit den Mitteln des Rechts im Sinne der Kommission korrigiert. Während eine jüngere Serie von EuGH-Urteilen zu ,Goldenen Aktien' ${ }^{31}$ das Gesellschaftsrecht stärker harmonisierte, als es durch die Übernahmerichtlinie gelungen war, weiteten die Urteile zu ,Laval', ,Rüffert' ${ }^{32}$ und ,Kommission gegen Luxemburg' ${ }^{33}$ das Herkunftslandprinzip bei der Arbeitnehmerentsendung aus, obwohl dieses Prinzip durch den Kompromiss bei der Dienstleistungsrichtlinie in weiten Bereichen dem arbeitsrechtlichen Territorialprinzip hatte weichen sollen. Auf weitere Neuerungen dieser Urteile wird unten noch einmal einzugehen sein. Doch bereits jetzt lässt sich festhalten, dass die Spielräume der Integration durch Recht in der jüngeren Vergangenheit nicht ab-, sondern eher zugenommen haben.

Ambivalenter erscheint die Beurteilung politischer Handlungsspielräume supranationaler Institutionen gegenüber potenziell integrationsunwilligen Mitgliedstaaten. Während die Verwässerung (Dienstleistungs- und Übernahmerichtlinie) oder das Scheitern einiger Richtlinien (Hafen- und Arbeitszeitrichtlinie) auf politische Blockaden im Zusammenspiel von Rat und Parlament verweisen, erkennt Kommissionspräsident José Manuel Barroso einen wachsenden Handlungsspielraum der Kommission. In einem Gespräch über zentrale politikwissenschaftliche Befunde der Integrationsforschung - zugrunde lagen unter anderem Texte von Hooghe und Marks, Moravcsik, Pollack und Scharpf - nennt Barroso zwei Folgen der Osterweiterung, die die Kommission aus seiner Sicht stärken. ${ }^{34}$ Erstens, die Koordinierungsfähigkeit der Mitgliedstaaten, Vorschläge der Kommission abzuändern, habe abgenommen. Gleichzeitig seien der Kommission, zweitens, mit der Osterweiterung natürliche Verbündete

29 Susanne K. Schmidt: Europäische Integration zwischen judikativer und legislativer Politik, in: Martin Höpner/ Armin Schäfer (Hrsg.): Die Politische Ökonomie der europäischen Integration, Frankfurt/New York 2008, S. 101-128.

30 Im Fall der Übernahmerichtlinie gelang es der Kommission nicht, eine strikte Verpflichtung auf die Neutralität des Managements im Fall feindlicher Übernahmeversuche sowie das Prinzip ,eine Aktie, eine Stimme ' durchzusetzen. Die Auseinandersetzungen um die Dienstleistungsrichtlinie endeten mit einem Kompromiss, der die Anwendung des umstrittenen Herkunftslandprinzips zunächst auf eng definierte Sachverhalte zu begrenzen schien.

31 Vgl. EuGH Rs. C-367/98 (Kommission/Portugal), Slg. 2002, I-4731; EuGH Rs. C-483/99 (Kommission/ Frankreich), Slg. 2002, I-4781; EuGH Rs. C-463/00 (Kommission/Spanien), Slg. 2003, I-4581. Noch nicht beendet ist in diesem Zusammenhang die Auseinandersetzung um das deutsche Volkswagengesetz. Vergleiche hierzu Peer Zumbansen/Daniel Saam: The ECJ, Volkswagen and European Corporate Law: Reshaping the European Varieties of Capitalism, Comparative Research in Law \& Political Economy: CLPE Research Paper $30 / 2007$.

32 EuGH Rs. C-346/06 (Rüffert), Slg. 2008, I-1989. Bei diesem Urteil ging es um die Europarechtswidrigkeit eines deutschen Vergabegesetzes, das die Zahlung ortsüblicher Entgelte zur Voraussetzung der Vergabe öffentlicher Aufträge machte.

33 EuGH Rs. C-319/06 (Kommission/Luxemburg), Slg. 2008, I-4323. Dieses Urteil richtete sich gegen Bestimmungen zur Inflationsindexierung von Mindestlöhnen im luxemburgischen Entsendegesetz.

34 Das Gespräch wurde von der „European Union Studies Association“ auf der EUSA-Konferenz in Montreal im Juli 2007 initiiert. John Peterson: Q and A with President Barroso, in: EUSA Review 4/2007, S. 1-5. 
bei ihrem Kampf für eine vertiefte Wirtschaftsintegration zur Seite gestellt worden. ${ }^{35}$ Kurz, einem eindeutigen Trend zur Intensivierung der ,Integration durch Recht" stehen widersprüchliche Befunde zu den Chancen der politisch herbeigeführten Wirtschaftsintegration gegenüber. Diesen Befund über die asymmetrischen Dynamiken unterschiedlicher Integrationsmodi in Rechnung gestellt, lässt sich nachfolgend betrachten, wie sich diese Entwicklungen auf unterschiedliche Dimensionen der Wirtschafts- und Sozialintegration auswirken.

\section{Drei Dimensionen der Wirtschafts- und Sozialintegration}

Die gegenwärtige Integrationsphase, so der Stand der bisherigen Argumentation, ist durch die Gleichzeitigkeit gegenläufiger Entwicklungen gekennzeichnet: Vertiefung der Integration durch die Nutzung der (vor allem: rechtlichen) Spielräume supranationaler Institutionen einerseits, höher gelegte Hürden intergouvernementaler Konsensfindung und effektiver Kontrolle aufgrund komplexer werdender Koordinationsprozesse und heterogener werdender politökonomischer Interessenlagen andererseits. Diese Entwicklungen berühren die Dynamiken der Wirtschafts- und Sozialintegration in unterschiedlicher Weise. ${ }^{36} \mathrm{Um}$ dies zu verdeutlichen, unterscheiden wir drei Dimensionen der Wirtschafts- und Sozialintegration: (1) marktgestaltende Integration, (2) marktschaffende Integration und (3) die Schaffung eines europäischen Antidiskriminierungsraums. Integrationsfortschritte sind in allen drei Dimensionen zu verzeichnen, deren Reichweiten und Geschwindigkeiten jedoch variieren.

\section{Marktgestaltende Integration}

In den Bereichen, in denen die Europäische Union sozialpolitische (hauptsächlich: arbeitsrechtliche) Kompetenzen besitzt und mit qualifizierter Mehrheit entscheiden kann, gelingt es immer wieder, gemeinsame Standards zu etablieren, die auch in entwickelten Sozialstaaten Veränderungsbedarf hervorrufen. Eine generelle Abkehr von Richtlinien als Politikinstrument zugunsten weicher Koordinationsformen lässt sich in diesem Bereich nicht feststellen. ${ }^{37}$ Auch marktbegleitende Vorschriften, wie die Verordnung (EWG) Nr. 1408/71 des Rates, ${ }^{38}$ sind mehrfach aktualisiert und erweitert worden. Mit dieser marktbegleitenden Sozialpolitik soll sichergestellt werden, dass mangelnde Transferierbarkeit sozialer Leistungen nicht die Mobilität im Binnenmarkt verhindert.

Seit Abschluss des Vertrags von Maastricht ist es allerdings nicht mehr gelungen, die Kompetenzen der Europäischen Union zur sozialpolitischen Marktgestaltung zu erweitern.

35 „Paradoxically, it is easier to take decisions now“, sagt Barroso, ,the role of the Commission is being reinforced now, with 27 [EU members], compared with previous years." Und: "[F]rom my own experience, the EU's institutions are far more autonomous than Institutionalist theory [...] would lead one to believe." Peterson: Q and A with President Barroso, 2007.

36 Grundlegend zu den unterschiedlichen Dynamiken negativer (in der Regel: marktschaffender) und positiver (in der Regel: marktkorrigierender) Integration: Fritz W. Scharpf: Negative and Positive Integration in the Political Economy of European Welfare States, in: Gary Marks/Fritz W. Scharpf/Philippe C. Schmitter/Wolfgang Streeck (Hrsg.): Governance in the European Union, London 1996, S. 15-39; Fritz W. Scharpf: Regieren in Europa. Effektiv und demokratisch?, Frankfurt/New York 1999; Fritz W. Scharpf: The European Social Model: Coping with the Challenges of Diversity, in: Journal of Common Market Studies 4/2002, S. 645-670.

37 Gerda Falkner/Oliver Treib/Miriam Hartlapp/Simone Leiber: Complying with Europe. EU Harmonisation and Soft Law in the Member States, Cambridge 2005, S. 41-55.

38 Verordnung (EWG) Nr. 1408/71 des Rates vom 14. Juni 1971 zur Anwendung der Systeme der sozialen Sicherheit auf Arbeitnehmer und deren Familien, die innerhalb der Gemeinschaft zu- und abwandern, in: Amtsblatt der EG, Nr. L 149 vom 5. Juli 1971, S. 2-50, zuletzt geändert durch Verordnung (EG) Nr. 883/2004 des Europäischen Parlaments und des Rates vom 29. April 2004 zur Koordinierung der Systeme der sozialen Sicherheit, in: Amtsblatt der EU, Nr. L 166 vom 30. April 2004, S. 1-123. 
Mit der Osterweiterung sind die Chancen auf eine Delegation sozialstaatlicher Umverteilungspraktiken von der nationalen auf die europäische Ebene weiter gesunken. ${ }^{39}$ Versuche der Vollharmonisierung drohen an den heterogenen Interessen der Mitgliedstaaten zu scheitern und werden von der Kommission deshalb nicht in Angriff genommen (oder auch nur für grundsätzlich sinnvoll erachtet). ${ }^{40}$ Nicht zuletzt aufgrund heterogener Sozialstaatstraditionen greifen die Regierungen zunehmend auf Formen, weicher' Koordinierung zurück. Nachdem zuerst 1997 mit der Europäischen Beschäftigungsstrategie eine Alternative zur Integration durch Richtlinien gefunden wurde, ist dieses Instrument unter dem Titel der, Offenen Methode der Koordinierung '(OMK) auf eine ganze Reihe von Politikfeldern übertragen worden. ${ }^{41}$ Kernelemente dieser Methode bestehen darin, zyklisch gemeinsame europäische Ziele zu definieren, die von den Mitgliedstaaten durch der nationalen Situation angemessene Maßnahmen umgesetzt werden sollen. Auf rechtlich verbindliche, sanktionierbare Standards wird bewusst verzichtet. Stattdessen sollen sich Einsichten in die transnationalen Folgen nationaler wirtschafts- und sozialpolitischer Entscheidungen, gemeinsame Problemperzeptionen und Reformanregungen aus wechselseitigem Austausch ergeben. Um Lernprozesse anzustoßen, berichtet die Kommission über die erzielten Fortschritte und es finden regelmäßig ,peer reviews' statt. ${ }^{42}$

Nach einer Phase der Proliferation und Zerfaserung der OMK wird seit einigen Jahren versucht, die Verfahren in der sogenannten Lissabon-Strategie zu bündeln. Strittig ist, welche Wirkung die OMK hat, vor allem, ob durch sie eine Politisierung europäischer und nationaler Politikgestaltung angestoßen wird. Während Zeitlin nur wenige Belege dafür findet, dass die OMK materielle Reformen angestoßen hat, hebt er hervor, dass sich Problemwahrnehmungen und politische Verfahren in den Mitgliedstaaten verändert haben. Er erkennt eine stärkere Öffnung des politischen Prozesses für zivilgesellschaftliche Gruppen. ${ }^{43} \mathrm{Im} \mathrm{Ge}-$ gensatz dazu dominiert in weiten Teilen der Literatur inzwischen eine skeptische Beurteilung des Aktivierungspotenzials der OMK. Einerseits wird die Tendenz zur Bürokratisierung und Professionalisierung der OMK hervorgehoben, die eine breitere Partizipation verhindere, andererseits die nur sporadische und oberflächliche Einbindung der Sozialpartner. ${ }^{44}$ Schließlich bleibt die öffentliche Wahrnehmung der OMK in den Mitgliedstaaten gering und hinter der Aufmerksamkeit zurück, die umstrittene Richtlinien und EuGH-Urteile erfahren haben.

39 Vergleiche beispielsweise Florian Rödl: Europäisches Verfassungsziel „,soziale Marktwirtschaft“ - kritische Anmerkungen zu einem populären Modell, in: integration 2/2005, S. 150-161.

40 So explizit auch Barroso. Peterson: Q and A with President Barroso, 2007.

41 Zur Entstehungsgeschichte siehe Michael W. Bauer/Ralf Knöll: Die Methode der offenen Koordinierung: Zukunft europäischer Politikgestaltung oder schleichende Zentralisierung?, in: Aus Politik und Zeitgeschichte B 1-2/2003, S. 33-38.

42 Zum Verfahren siehe Jonathan Zeitlin: The Open Method of Coordination in Question, in: Jonathan Zeitlin/ Philippe Pochet (Hrsg.): The Open Method of Coordination in Action. The European Employment and Social Inclusion Strategies, Brüssel 2005, S. 19-36; Armin Schäfer: Die neue Unverbindlichkeit. Wirtschaftspolitische Koordinierung in Europa, Frankfurt 2005.

43 Jonathan Zeitlin: The Open Method of Coordination and Reform of National Social and Employment Policies: Influences, Mechanisms, Effects, in: Martin Heidenreich/Jonathan Zeitlin (Hrsg.): Changing European Employment and Welfare Regimes. The Influence of the Open Method of Coordination on National Reforms, London 2009, S. 214-237.

44 Martin Heidenreich/Gabriele Bischoff: Die offene Methode der Koordinierung: Ein europäisches Instrument zur Modernisierung nationaler Sozial- und Beschäftigungsordnungen?, in: Martin Heidenreich (Hrsg.): Die Europäisierung sozialer Ungleichheit, Frankfurt/New York 2006, S. 277-311; Caroline de la Porte/Philippe Pochet: Participation in the Open Method of Coordination. The Cases of Employment and Social Inclusion, in: Jonathan Zeitlin/Philippe Pochet (Hrsg.): The Open Method of Coordination in Action. The European Employment and Social Inclusion Strategies, Brüssel 2005, S. 353-390. 
Neben dem Rückgriff auf unverbindliche Koordinierungsverfahren liegt eine weitere Innovation europäischer Politik in der Aufwertung des Sozialen Dialogs. Den europäischen Sozialpartnern - Gewerkschaften, Arbeitgebern und Industrieverbänden - wurde mit dem Vertrag von Maastricht die Möglichkeit eingeräumt, Richtlinienvorschläge auszuhandeln, die durch den Ministerrat Verbindlichkeit erhalten. In den 1990er Jahren sind auf diesem Wege mehrere sogenannte Sozialpartner-Richtlinien verabschiedet worden. ${ }^{45}$ Doch seit der Jahrtausendwende werden diese Abkommen durch an die OMK angelehnte, rechtlich nicht bindende und sektorenspezifische Formen der Koordinierung verdrängt. Nach anfänglichen Erfolgen sieht es nun nicht mehr so aus, als könne es gelingen, die sozialpolitische Schwerfälligkeit des Ministerrats durch Initiativen der Sozialpartner zu überwinden. ${ }^{46}$ Die ohnehin nur bedingt verpflichtungsfähigen europäischen Dachverbände stehen, wie auch die Politik, nach der Osterweiterung vor der Herausforderung, heterogene Ausgangslagen und Interessen aufeinander abstimmen zu müssen. Eine einheitliche marktgestaltende Politik der Europäischen Union ist unter diesen Umständen weder vom Ministerrat noch von den Sozialpartnern zu erwarten.

\section{Marktschaffende Integration}

Im Gegensatz zur Blockadeanfälligkeit einer supranationalen Sozialpolitik eignet sich die zunehmende Schärfung europarechtlicher Instrumente für eine stetige Vertiefung der marktschaffenden Integration. Insbesondere Scharpf hatte in seiner Theorie einer verfestigten Asymmetrie zwischen negativer und positiver Integration auf die Anwendung des europäischen Wettbewerbsrechts auf Sektoren hingewiesen, für die dieses Recht ursprünglich nicht gedacht war: auf die staatsnahen Bereiche öffentlicher Daseinsvorsorge wie Telekommunikation, Energie und Transport. ${ }^{47}$ Die Erschließung neuer Anwendungsbereiche des europäischen Wettbewerbsrechts schreitet auch in der gegenwärtigen Integrationsphase voran. Aktuelle Konflikte betreffen beispielsweise das öffentliche Bankenwesen, den öffentlichrechtlichen Rundfunk und das Krankenhauswesen. Ein besonders hervortretendes Merkmal der jüngeren Integrationsgeschichte besteht jedoch vor allem in überraschend extensiven Interpretationen der Binnenmarktfreiheiten durch Kommission und EuGH und deren Anwendung auf Regulierungsfelder, die zuvor ausschließlich den Mitgliedstaaten oblagen. ${ }^{48}$

Ein Beispiel hierfür ist die vom EuGH angestoßene Liberalisierung des Gesellschaftsrechts. ${ }^{49}$ Bis in die jüngere Vergangenheit hinein galt als ausgemacht, dass das Europarecht der Anwendung der sogenannten Sitztheorie nicht entgegensteht. Sie besagt, dass sich die Rechtsverhältnisse einer Gesellschaft nicht nach dem Ort ihrer Gründung, sondern nach dem Ort des tatsächlichen Firmensitzes richten. Befand sich der Sitz eines Unternehmens also in

45 Vgl. Gerda Falkner: EU Social Policy in the 1990s: Towards a Corporatist Policy Community, London 1998.

46 Stijn Smismans: The European Social Dialogue in the Shadow of Hierarchy, in: Journal of Public Policy 1/2008, S. 161-180; Armin Schäfer/Simone Leiber: The Double Voluntarism in EU Social Dialogue and Employment Policy, in: Sandra Kröger (Hrsg.): What We Have Learnt: Advances, Pitfalls and Remaining Questions in OMC Research, European Integration online Papers, Sonderheft 1/2009.

47 Scharpf: Regieren in Europa, 1999, S. 47-80.

48 Auf dieses Problem weisen auch jene Autoren hin, denen es eigentlich um den Schutz der Kommission und des EuGH vor der Kritik der Integrationsskeptiker geht. So schreibt beispielsweise Franz C. Mayer, die Frage sei zu stellen, „ob die durch den EuGH entwickelte Rechtsstruktur im Bereich der Grundfreiheiten sich nicht zu sehr von dem, worum es eigentlich bei Grundfreiheiten und Binnenmarkt gehen sollte, entfernt hat." Franz C. Mayer: Der EuGH als Feind? Die Debatte um das soziale Europa in der europäischen Rechtsprechung, in: integration 3/2009, S. 246-265.

49 Siehe hierzu beispielsweise Silja Maul/Claudia Schmidt: Inspire Art: Quo vadis Sitztheorie?, in: Betriebs-Berater 44/2003, S. 2297-2300; Christoph Binge/Ulrich Thölke: Everything goes: Das deutsche Internationale Gesellschaftsrecht nach Inspire Art, in: Deutsche Notar-Zeitschrift 1/2004, S. 21-33. 
Deutschland, erlangte es nur formalen Unternehmensstatus, wenn es im Einklang mit dem deutschen Gesellschaftsrecht registriert wurde. Die Anwendung dieser Sitztheorie verwarf der EuGH mit seinen Urteilen zu ,Centros', ${ }^{50}$,Überseering, ${ }^{51}$ und ,Inspire Art ${ }^{6} .{ }^{52}$ Sie behindere, so urteilte das Gericht, die Niederlassungsfreiheit über Gebühr und sei nicht von zwingenden Gründen des Allgemeininteresses gedeckt. Von besonderer Brisanz ist dabei, dass auch die Gründung ausländischer Briefkastenfirmen (Firmensitze ohne praktische Bedeutung für die wirtschaftlichen Aktivitäten) vom EuGH nicht als illegitimer Umgehungstatbestand, sondern als vom Europarecht getragene Ausübung der Marktfreiheiten angesehen wurde. Das bedeutet konkret, dass für Unternehmensgründer nunmehr eine faktische Wahlfreiheit der in den EU-27 vertretenen Rechtsformen besteht. ${ }^{53}$ Wachsen diese Unternehmen über die Schwelle von 500 beziehungsweise 2.000 Beschäftigten, greift keine deutsche Arbeitnehmermitbestimmung auf Ebene der Leitungsorgane. ${ }^{54}$ In der Tat haben diese Urteile zu einem Boom von in Deutschland aktiven ausländischen Rechtsformen geführt, darunter auch mehrere mitbestimmungsrelevante Fälle. ${ }^{55}$

Besondere Aufmerksamkeit haben jüngst die bereits erwähnten Urteile zu ,Viking ‘, ,Laval' und ,Rüffert' auf sich gezogen. Bei diesen Urteilen überraschte der EuGH unter anderem mit einer bemerkenswerten Ausdehnung der Verpflichtung Privater durch die europäischen Grundfreiheiten. Demnach verpflichten die Grundfreiheiten Gewerkschaften, so das Urteil im Fall ,Laval', im Falle der Behinderung transnationaler wirtschaftlicher Betätigung durch Arbeitskämpfe nachzuweisen, dass diese verhältnismäßig und die mit ihnen verfolgten Ziele durch zwingende Gründe des Allgemeininteresses gerechtfertigt sind. Im Fall ,Rüffert` erklärte der EuGH öffentliche Ausschreibungen, bei denen die Zahlung ortsüblicher Löhne zur Voraussetzung der Auftragsvergabe gemacht wurde, zu Verstößen gegen die Dienstleistungsfreiheit. ${ }^{56}$ In einer anderen, ebenfalls oben bereits erwähnten Urteilsserie prüfte der EuGH staatliche Sonderstimmrechte bei privatisierten Unternehmen (,Goldene Aktien") gegen die Kapitalverkehrsfreiheit und kam nahezu immer zu dem Schluss, dass die Sonderstimmrechte gegen europäisches Recht verstießen. Noch während den Verhandlungen über die Übernahmerichtlinie war angenommen worden, dass solche Regelungen nur auf politischem Wege abgeschafft werden könnten.

Erwähnenswert und von großer praktischer Brisanz sind zudem jüngere Urteile zum europäischen Steuerwettbewerb, in denen der EuGH Praktiken der Steuervermeidung als von der Binnenmarktlogik getragen legitimierte, jedoch Versuche nationaler Regierungen, diese

50 EuGH Rs. C-212/97 (Centros), Slg. 1999, I-1459.

51 EuGH Rs. C-208/00 (Überseering), Slg. 2002, I-9919.

52 EuGH Rs. C-167/01 (Inspire Art), Slg. 2003, I-10155.

53 Damit nähert der EuGH das europäische Recht an die amerikanische Situation an: In den USA werden viele Unternehmen wegen der dortigen vergleichsweise laxen gesellschaftsrechtlichen Standards im Staat Delaware gegründet. Vergleiche hierzu Mark J. Roe: Delaware's Politics, in: Harvard Law Review 3/2005, S. 24932543 .

54 Speziell zu den Implikationen für die Arbeitnehmermitbestimmung: Marcus Kamp: Die unternehmerische Mitbestimmung nach 'Überseering' und 'Inspire Art', in: Betriebs-Berater 27/2004, S. 1496-1500. Unklar ist bisher, ob eine etwaige verpflichtende Anwendung der Mitbestimmung auf ausländische Rechtsformen dem Europarecht standhalten würde. Vergleiche zu dieser Diskussion Otto Sandrock: Gehören die deutschen Regelungen über die Mitbestimmung auf Unternehmensebene wirklich zum deutschen ordre public?, in: Die Aktiengesellschaft 2/2004, S. 57-66.

55 Vergleiche im Einzelnen Sebastian Sick: Mitbestimmungsrelevante Unternehmen mit ausländischen/kombiniert ausländischen Rechtsformen, Düsseldorf 2008, abrufbar unter: http://www.boeckler.de/pdf/mbf_2008_06 _19_sick.pdf (letzter Zugriff: 19.12.2009). Der Autor zählt von Dezember 2006 bis Juni 2008 eine Zunahme mitbestimmungsrelevanter Fälle (über 500 Arbeitnehmer) von 17 auf 29.

56 Stellvertretend für zahlreiche kritische Besprechungen dieser Urteile: Christian Joerges/Florian Rödl: Das soziale Defizit des Europäischen Integrationsprojekts, in: Kritische Justiz 2/2008, S. 149-165. 
einzudämmen, als nicht von „Zwingenden Gründen des Allgemeininteresses“ gedeckt verwarf. Durch diese Rechtsprechung verschärft der EuGH den innereuropäischen Steuerwettbewerb, ${ }^{57}$ während sich die Mitgliedstaaten aufgrund divergierender Interessen nicht darauf einigen können, den Wettbewerb politisch einzudämmen. Kurz: In der jüngeren Integrationsphase wurden außerordentliche Fortschritte der Marktintegration durch juristische Prüfung nationaler, sehr heterogener Regelungsbestände gegen die europäischen Grundfreiheiten erzielt. Einige dieser Entscheidungen haben eine starke Politisierung in den betroffenen Mitgliedstaaten hervorgerufen. ${ }^{58}$

\section{Schaffung eines europäischen Antidiskriminierungsraums}

Fortschritte wurden jüngst in einem dritten Politikfeld erreicht, das quer zu wirtschaftlicher Liberalisierung einerseits und europäischer Sozialpolitik andererseits steht: der Schaffung eines europäischen Antidiskriminierungsraums. ${ }^{59}$ Verbesserungen des Schutzes vor Diskriminierungen aufgrund des Geschlechts, des Alters, sexueller Orientierungen sowie der Zugehörigkeit zu Ethnien und Religionen gehen in vielen Mitgliedstaaten auf die Europäische Union zurück. Das gilt insbesondere für den Schutz vor diskriminierenden Behinderungen des Zugangs zu Arbeitsmärkten und vor Diskriminierungen am Arbeitsplatz. Für diese Erfolge sind sowohl die europäische Politik als auch die richterliche Fortentwicklung des europäischen Rechts verantwortlich. So ist dem politischen Verhandlungsgeschick der Kommission die Verabschiedung von vier Antidiskriminierungsrichtlinien in den Jahren 2000 bis $2004 \mathrm{zu}$ verdanken, ${ }^{60}$ deren Umsetzung in Deutschland mit dem Allgemeinen Gleichbehandlungsgesetz von 2006 erfolgte.

Auch wenn die relative Bedeutung politischer Übereinkünfte bei der europäischen Antidiskriminierungspolitik höher einzuschätzen ist als im Fall der oben skizzierten Maßnahmen marktschaffender Politik, darf gleichwohl der große Anteil des Richterrechts an der Formulierung europäischer Gleichbehandlungspolitik nicht unterschätzt werden. So gelang es dem EuGH, auf Grundlage vergleichsweise schmaler Bestimmungen in den europäischen Verträgen - vor allem: dem Grundsatz der Lohngleichheit der Geschlechter und der Arbeitnehmerfreizügigkeit - eine extensive Antidiskriminierungsjudikatur zu entwickeln, die konkrete Auswirkungen in allen Mitgliedstaaten entfaltete. Herausragende Beispiele für die jüngere Rechtsprechung sind das umstrittene ,Mangold'-Urteil, mit dem der EuGH die erleichterte Befristung von Arbeitsverträgen älterer Arbeitnehmer in Deutschland aushebelte; ${ }^{61}$ das , Ma-

57 Stellvertretend für andere: EuGH Rs. C-446/03 (Marks \& Spencer), Slg. 2005, I-10837. Heinemann und Wendt sprechen von einem ,bemerkenswerten Rigorismus“ der Steuerrechtsprechung des EuGH, Drüen und Kahler nennen sie ,strukturell destruktiv“. Siehe Friedrich Heinemann/Carsten Wendt: EU-Steuerharmonisierung auf dem Gebiet der Unternehmensbesteuerung - Stand und Perspektiven, in: integration 3/2007, S. 281291, hier S. 283 sowie Klaus-Dieter Drüen/Björn Kahler: Die nationale Steuerhoheit im Prozess der Europäisierung, in: Steuern und Wirtschaft 2/2005, S. 171-184, hier S. 183. Vergleiche allgemein zum europäischen Steuerwettbewerb Steffen Ganghof/Philipp Genschel: Deregulierte Steuerpolitik: Körperschaftssteuerwettbewerb und Einkommensbesteuerung in Europa, in: Martin Höpner/Armin Schäfer (Hrsg.): Die Politische Ökonomie der europäischen Integration, Frankfurt/New York 2008, S. 311-334.

58 Besonders stark gilt dies für die Reaktionen auf die ,Laval'-Entscheidung in Schweden.

59 Grundlegend Evelyn Ellis: EU Anti-Discrimination Law, Oxford 2005.

60 Richtlinie 2000/43/EG des Rates vom 29. Juni 2000 zur Anwendung des Gleichbehandlungsgrundsatzes ohne Unterschied der Rasse oder der ethnischen Herkunft, in: Amtsblatt der EU, Nr. L 180 vom 19. Juli 2000, S. 2226; Richtlinie 2000/78/EG des Rates vom 27. November 2000 zur Festlegung eines allgemeinen Rahmens für die Verwirklichung der Gleichbehandlung in Beschäftigung und Beruf, in: Amtsblatt der EU, Nr. L 303 vom 2. Dezember 2000, S. 16-22; Richtlinie 2004/113/EG des Rates vom 13. Dezember 2004 zur Verwirklichung des Grundsatzes der Gleichbehandlung von Männern und Frauen beim Zugang zu und bei der Versorgung mit Gütern und Dienstleistungen, in: Amtsblatt der EU, Nr. L 373 vom 21. Dezember 2004, S. 37-43.

61 EuGH Rs. C-144/04 (Mangold), Slg. 2005, I-9981. 
ruko'-Urteil, das sich gegen die Verweigerung von Hinterbliebenenrenten bei homosexuellen Partnern richtete; ${ }^{62}$ die ,Coleman'-Entscheidung, mit der der EuGH Diskriminierungsverbote am Arbeitsplatz auch auf Angehörige der durch das europäische Recht geschützten Arbeitnehmer ausweitete; ${ }^{63}$ und jüngst das ,Feryn'-Urteil, mit dem der EuGH den Schutz vor Diskriminierungen auch auf abstrakte Äußerungen von Arbeitgebern anwandte, die keine sichtbare Handlungskonsequenz entfalten. ${ }^{64}$ Eine eigenständige Serie von Antidiskriminierungsurteilen betrifft den transnationalen Zugang zu den sozialen Sicherungssystemen der Mitgliedstaaten. ${ }^{65}$

Waren soziale Rechte in der Europäischen Union lange Zeit ausschließlich auf Arbeitnehmer bezogen, hat der EuGH sie nach und nach aus dieser häufig kritisierten Beschränkung befreit. An die Stelle der Rechte von Marktbürgern treten die der Unionsbürger. Dieser Übergang gelingt dem EuGH durch die Verbindung von Freizügigkeit (Art. 21 AEUV) und dem Verbot der Diskriminierung aus Gründen der Staatsangehörigkeit (Art. 18 AEUV). Jeder Unionsbürger - das sind Personen, die Staatsangehörige eines Mitgliedstaates sind -, der sich rechtmäßig in einem Mitgliedsland aufhält, darf dort nicht anders als die Inländer behandelt werden. Unionsbürger haben nach dieser Rechtsprechung auch in anderen als ihren Herkunftsländern prinzipiell Anspruch auf soziale Leistungen, selbst wenn sie keinen Beitrag zu deren Finanzierung leisten. ${ }^{66}$ Im ,Grzelczyk'-Urteil betont der EuGH, dass sich aus dem Recht der Gemeinschaft das Erfordernis finanzieller Solidarität der Angehörigen eines Staates mit denen anderer Mitgliedstaaten ergibt, solange dies die öffentlichen Finanzen des Gastlandes nicht über Gebühr belastet. ${ }^{67}$ Mit dieser Erweiterung sozialer Rechte enteilt der Gerichtshof der von den Mitgliedstaaten bislang akzeptierten Solidaritätsnorm, die das Aufenthaltsrecht daran knüpft, dass während des Aufenthalts keine Sozialhilfeleistungen in Anspruch genommen werden und ein umfassender Krankenversicherungsschutz vorliegt. ${ }^{68}$

Kennzeichnend für die neuere Rechtsprechung ist, dass die politisch restriktiv definierten Aufenthaltsbestimmungen aufgeweicht werden und eine Ausweitung individueller Rechte stattfindet. Obwohl Art. 20 AEUV von Rechten und Pflichten spricht, die aus der Unionsbürgerschaft folgen, besteht kaum die Möglichkeit, denjenigen Pflichten aufzuerlegen, die von neu gewonnenen sozialen Rechten profitieren. Der Zusammenhang von Beitragspflichten und Anspruchsrechten wird entkoppelt und eine anspruchsvolle Solidaritätsnorm entwickelt: „Indem der EuGH zunehmend Sozialleistungen transnational zugänglich macht“, so stellt Haltern fest, ,stellt er finanzielle Anforderungen, die nur dann erträglich sind, wenn

62 EuGH Rs. C-267/06 (Maruko), Slg. 2008, I-1757.

63 EuGH Rs. C-303/06 (Coleman), Slg. 2008, I-5603.

64 EuGH Rs. C-54/07 (Feryn), Slg. 2008, I-5187.

65 Vergleiche hierzu die Diskussion bei Fritz W. Scharpf: Individualrechte gegen nationale Solidarität, in: Martin Höpner/Armin Schäfer (Hrsg.): Die Politische Ökonomie der europäischen Integration, Frankfurt/New York 2008, S. 89-99.

66 EuGH Rs. C-85/96 (Martínez Sala), Slg. 1998, I-2691; EuGH Rs. C-184/99 (Grzelczyk), Slg. 2001, I-6193; EuGH Rs. C-224/98 (D’Hoop), Slg. 2002, I-6191; EuGH Rs. C-413/99 (Baumbast), Slg. 2002, I-7091; EuGH Rs. C-456/02 (Trojani), Slg. 2004, I-7573; EuGH Rs. C-138/02 (Collins), Slg. 2004, I-2703; EuGH Rs. C-209/ 03 (Bidar), Slg. 2005, I-2119. Siehe zu den sozialen Rechten, die aus der Rechtsprechung folgen: Thomas Kingreen: Die Universalisierung sozialer Rechte im europäischen Gemeinschaftsrecht, in: Armin Hatje/Peter M. Huber (Hrsg.): Europarecht, Beiheft 1: Unionsbürgerschaft und soziale Rechte, Baden-Baden 2007, S. 43-74.

67 EuGH Rs. C-184/99 (Grzelczyk), Slg. 2001, I-6193, Rn. 44.

68 So Art. 7 der Richtlinie 2004/38/EG des Europäischen Parlaments und des Rates vom 29. April 2004 über das Recht der Unionsbürger und ihrer Familienangehörigen, sich im Hoheitsgebiet der Mitgliedstaaten frei zu bewegen und aufzuhalten, zur Änderung der Verordnung (EWG) Nr. 1612/68 und zur Aufhebung der Richtlinien 64/221/EWG, 68/360/EWG, 72/194/EWG, 73/148/EWG, 75/34/EWG, 75/35/EWG, 90/364/EWG, 90/365/ EWG und 93/96/EWG, in: Amtsblatt der EU, Nr. L 158 vom 30. April 2004, S. 77-123. Kritisch zu dieser Rechtsprechung: Kay Hailbronner: Die Unionsbürgerschaft und das Ende rationaler Jurisprudenz durch den EuGH?, in: Neue Juristische Wochenschrift 31/2004, S. 2185-2189. 
auch eine kollektive Dimension von Solidarität und letztlich Zugehörigkeit existiert“ “69 Damit unterstellt der Europäische Gerichtshof, dass eine kollektive europäische Identität vorhanden ist, die - wie Offe einmal formulierte - „Umverteilungszumutungen“70 möglich macht. Angesichts der bereits genannten Konflikte zwischen und der Ängste in den Mitgliedstaaten trägt diese kontrafaktische Unterstellung die Gefahr negativer Politisierung in sich.

\section{Fazit und Ausblick: warum weniger mehr sein könnte}

In diesem Text haben wir den gegenwärtigen Stand der Wirtschaftsintegration untersucht. Als entscheidende Neuerungen der jüngeren Integrationsphase wurden zum einen die gewachsene Heterogenität von Wohlstand sowie Produktions- und Verteilungskulturen sowie zum anderen eine sich beschleunigende Bereitschaft zur kreativen, extensiven Anwendung des Wettbewerbsrechts, der Grundfreiheiten sowie europäischer Diskriminierungsverbote herausgestellt. Die gestiegene Heterogenität vermindert sowohl die Wahrscheinlichkeit einer mittelfristigen Supranationalisierung von Sozialstaatlichkeit als auch den koordinierten Widerspruch gegen marktschaffende Maßnahmen, die durch extensive, oft überraschende Auslegungen der europäischen Grundfreiheiten sowie des Wettbewerbsrechts durch Kommission und EuGH zustande kommen. Angesichts der Gleichzeitigkeit von heterogener werdenden politökonomischen Interessenlagen und der judikativen Fortentwicklung europäischen Rechts verfestigt sich die Asymmetrie zwischen der Beseitigung von (vermeintlichen) Binnenmarkthindernissen auf nationalstaatlicher Ebene einerseits und marktkorrigierender Integration auf europäischer Ebene andererseits. Die institutionalisierte Koordinierung der nationalen Arbeits- und Sozialverfassungen gewinnt zwar mit der OMK an Bedeutung, ist aber weit davon entfernt, im Europa der 27 auf einheitliche Standards hinwirken zu können oder auch nur zu wollen.

Allerdings existiert eine dritte, höchst erfolgreiche und sich beschleunigende Dimension der politökonomischen Integration, die sowohl auf europäischen Diskriminierungsverboten als auch auf der Arbeitnehmerfreizügigkeit aufbaut: die Antidiskriminierungspolitik und die mit ihr einhergehende, äußerst kraftvolle Rechtsprechung. Ihre Schwerpunkte liegen im diskriminierungsfreien Zugang zu Arbeitsmärkten, in der Nichtdiskriminierung am Arbeitsplatz und im transnationalen Zugang zu den sozialen Sicherungssystemen der Mitgliedstaaten. Wäre diese Dimension nicht als funktionales Äquivalent zur gebremsten Entwicklung eines europäischen Sozialstaats anzusehen, die die strukturelle Asymmetrie von marktschaffender und marktgestaltender Integration partiell aufhebt?

In Abwägung der widersprüchlichen Wirkungen europäischer Antidiskriminierungspolitik wird diese Frage letztlich verneint werden müssen. Freilich: Ihr Erfolg markiert einen Modernisierungsschub, der ohne die europäische Integration undenkbar wäre. Sie ist zudem ein Bereich europäischer Politik, der für die Bürgerinnen und Bürger direkt erfahrbar und als Ausfluss europäischer Politik identifizierbar ist. Aber: Sie ist kein Bestandteil und auch kein Substitut einer marktkorrigierenden Politik auf europäischer Ebene. Sie ist vielmehr ein Spezialfall von Chancengleichheitspolitik: ${ }^{71}$ Sie zielt auf freien, von Diskriminierungen nicht gestörten Marktzugang. Als solche ist sie von der Etablierung von Solidargemeinschaften

69 Ulrich Haltern: Europarecht und das Politische, Tübingen 2005, S. 493.

70 Offe: Demokratie und Wohlfahrtsstaat, 1998, S. 133.

71 Siehe ausführlich Alexander Somek: Das europäische Sozialmodell: Diskriminierungsschutz und Wettbewerb, in: juridikum 3/2008, S. 118-125, hier S. 118-120. Der Autor betont insbesondere die grundsätzliche Kompatibilität von marktschaffender Politik einerseits und auf Chancengleichheit zielender Antidiskriminierungspolitik andererseits. 
auf europäischer Ebene strikt zu unterscheiden. Sie steht quer zur Unterscheidung von marktschaffender und marktkorrigierender Politik, weil sie vor allem marktbefähigende Züge trägt. Chancengleichheitspolitik als Antidiskriminierungspolitik zielt auf freie, aber faire Märkte. Die Rechtsprechung zur Unionsbürgerschaft geht einen Schritt weiter und entkoppelt soziale Rechte vom Status des Marktbürgers. Hier entsteht eine nicht mehr bloß, akzessorische' Sozialpolitik, die primär die Funktionsweise der Märkte fördern soll. Allerdings unterstellt diese Rechtsprechung, dass bereits ein Zusammengehörigkeitsgefühl der Unionsbürger besteht, das grenzüberschreitende Solidarität tragen kann.

Wie wirken diese Entwicklungen auf die Chancen einer vertieften politischen Integration? Zwei Beobachtungen sind zur Beantwortung dieser Frage hilfreich. Die erste Beobachtung ist die einer nachhaltigen, sich über unterschiedliche Politikfelder erstreckenden (negativen) Politisierung europäischer Initiativen und Entscheidungen. Seit den 1990er Jahren ist die Wirtschaftsintegration wiederholt Gegenstand öffentlicher Auseinandersetzungen mit breiter öffentlicher Wahrnehmung geworden, vom Protest gegen die Übernahme- oder die Hafenrichtlinie bis hin zu einem neuartigen Grad an Aufmerksamkeit gegenüber wirtschafts- und sozialpolitisch relevanten Urteilen des EuGH. ${ }^{72}$ Besonders hervorzuheben ist in diesem Zusammenhang die transnationale Diskussion der Dienstleistungsrichtlinie. Im Zuge dieser Auseinandersetzung wurde - in den Worten von van de Steeg und Risse ${ }^{73}$ - wohl erstmals ${ }^{74}$ ein europäisches Thema in praktisch allen Mitgliedstaaten zur selben Zeit mit großer Vehemenz und vergleichbaren Relevanzkriterien diskutiert. Die Integration wird immer weniger als unpolitisch-technische Angelegenheit begriffen und durch einen außenpolitischen Konsens getragen. Diese Entwicklung ist vor allem als Folge der zugenommenen Integrationstiefe zu deuten.

Die Implikationen für die politische Integration sind bislang weniger vorteilhaft als von jenen erhofft, die in der Europäisierung nationaler Öffentlichkeiten eine Vorstufe einer politischen Gemeinschaft sehen. Einerseits erschwert die Politisierung europäischer Entscheidungen den Integrationsprozess, stellt dessen Elitenorientierung infrage und legt politischen Integrationsprojekten höhere Hürden auf. Gleichzeitig aber betonen Teilnehmer der Debatte um das europäische Demokratiedefizit die Chance, dass die Europäische Union in dem Maße Demokratiereife erlangt, in dem europäische Themen nicht länger exklusiv aus nationaler Perspektive diskutiert werden, sondern sich länderübergreifend ähnliche Diskursmuster nachweisen lassen. ${ }^{75}$ Aus transnational geteilten Perzeptionen könnte eine europäisierte Öffentlichkeit mit einem europäischen Parteiensystem entstehen, ${ }^{76}$ deren Abwesenheit bislang als eine Ursache für das europäische Demokratiedefizit identifiziert wurde. Geschieht dies, ist der Grundstein für eine kollektive Identität der Europäer gelegt.

Allerdings, und das ist unsere zweite Beobachtung, sortierten sich die Auseinandersetzungen über die jüngeren, hoch politisierten Integrationsprojekte weniger entlang einer

72 In diesem Zusammenhang erscheint konsequent, dass die allgemeine Zustimmung zur Europäischen Union in einigen ihrer Gründerstaaten, so Belgien, Frankreich und Deutschland, in den 1990er Jahren spürbar abnahm. Siehe Guido Tiemann: Sozioökonomische Determinanten von Euroskeptizismus und Integrationsunterstützung, in: Martin Höpner/Armin Schäfer (Hrsg.): Die Politische Ökonomie der europäischen Integration, Frankfurt/New York 2008, S. 241-276, hier S. 246-248.

73 Marianne van de Steeg/Thomas Risse: The Emergence of a European Community of Communication: Insights from Empirical Research on the Europeanization of Public Spheres, unveröffentlichtes Manuskript, 2007.

74 Hiervon auszunehmen sind Debatten über institutionelle Reformen wie die Schaffung der Wirtschafts- und Währungsunion. Ähnliches gilt für Debatten über Erweiterungsrunden.

75 Van de Steeg/Risse: The Emergence of a European Community, 2007.

76 Diese Hoffnung äußert beispielsweise Jo Leinen: Europäische Parteien: Aufbruch in eine neue demokratische EU, in: integration 3/2006, S. 229-235. 
transnationalen Links-rechts-Achse, als vielmehr entlang nationaler Interessen zur Verteidigung von Produktions- und Verteilungskulturen. Das galt für die Übernahmerichtlinie wie auch für die Dienstleistungsrichtlinie, ${ }^{77}$ die stellvertretend für zwei entscheidende Baustellen marktschaffender Integration stehen, nämlich für die europäische Organisation der ,fiktiven“ (Polanyi) Märkte für Kapital und Arbeit. Im Fall der Übernahmerichtlinie verlief die dominante Konfliktlinie zwischen den koordinierten und marktliberalen europäischen Spielarten des Kapitalismus, im Fall der Dienstleistungsrichtlinie standen Großbritannien und die osteuropäischen Beitrittsländer gegen die kontinentaleuropäischen und skandinavischen Mitgliedstaaten. ${ }^{78}$ Diese politische Dynamik ist keine historische Zufälligkeit, sondern ein systematischer Effekt. Sie wurzelt in einer Vertiefung der Wirtschaftsintegration, die sich gegenüber den europäischen Spielarten des Kapitalismus und ihren Wohlstandsunterschieden nicht länger neutral verhält.

Eine solche Neutralität der Wirtschaftsintegration, so ließe sich einwenden, hat es freilich nie gegeben. Stets waren die nationalen wirtschaftlichen Interessen an Vertiefungen der Integration unterschiedlich gelagert. Gleichwohl haben sich Gegenstand und Reichweite der Integration verändert. Bis in die 1990er Jahre hinein stand die Verwirklichung des Binnenmarkts für Güter im Vordergrund. Sie verschärfte den Wettbewerb zwischen den europäischen Spielarten des Kapitalismus, ließ deren Institutionen aber im Wesentlichen unberührt. Sie ließ Raum für verschiedene Strategien, Wettbewerbsvorteile zu erlangen, die institutionell höchst unterschiedlich unterfüttert waren und dies im Prinzip auch bleiben konnten, von der marktliberalen Wirtschaftsorganisation Großbritanniens über das organisierte Modell Deutschlands und das etatistische Modell Frankreichs bis hin zur skandinavischen Spielart des Kapitalismus. Die nunmehr im Vordergrund stehende Integration der Kapital- und Arbeitsmärkte ruft hingegen asymmetrische Anpassungslasten hervor. So wie die versuchte Schaffung eines einheitlichen europäischen Markts für freundliche und feindliche Unternehmensübernahmen für Frankreich institutionell etwas anderes bedeutet als für Großbritannien, hat die Öffnung der Dienstleistungsmärkte für Hochlohnökonomien wie Deutschland andere Implikationen als für die osteuropäischen Beitrittsländer. In beiden Fällen definierten die Akteure ihre Interessen zuvörderst als länderbezogene, parteien- und klassenübergreifende Interessen. Aus der Politisierung solcher Konstellationen, darauf haben Autoren wie Bartolini hingewiesen, ${ }^{79}$ entsteht eine ,Politisierungsgemeinschaft ${ }^{6}$, aber kein gemeinsamer politischer Raum oder eine kollektive Identität.

77 Diese ,Clash of Capitalisms'-Konstellation war im Fall der Übernahmerichtlinie deutlicher als im Fall der Dienstleistungsrichtlinie. Deutlich erkennbar war sie aber auch bei letzterer. Vehemente Proteste gegen den ursprünglichen Kommissionsvorschlag vom Januar 2004 wurden vor allem in den organisierten Ökonomien Kontinental- und Nordeuropas laut. Obwohl der schließlich erzielte Kompromiss auf einer Einigung zwischen der bürgerlich-konservativen und der sozialistischen Fraktion des Europäischen Parlaments beruhte, befürworteten Teile der Mitte-rechts-Fraktionen aus Großbritannien, Spanien, Polen, Ungarn und der Tschechischen Republik eine tiefgreifendere Liberalisierung der Dienstleistungsmärkte.

78 Dies lässt sich besonders eindrücklich anhand von namentlichen Abstimmungen im Europäischen Parlament demonstrieren. Mithilfe von Regressionstechniken lässt sich analysieren, ob die Parteien- oder die Länderzugehörigkeit der Abgeordneten stärkere Prädiktoren des Abstimmungsverhaltens waren. Siehe hierzu beispielsweise Helen Callaghan/Martin Höpner: European Integration and the Clash of Capitalisms. Political Cleavages over Takeover Liberalization, in: Comparative European Politics 3/2005, S. 307-332; Nils Ringe: Policy Preference Formation in Legislative Politics: Structures, Actors, and Focal Points, in: American Journal of Political Science 4/2005, S. 731-745.

79 Die ,transformation of territorial conflicts into partisan and cross-national ones“, so Bartolini, könne im Zuge zunehmender Politisierung europäischer Entscheidungen nicht unbesehen vorausgesetzt werden; erfolge sie nicht, würden die Gefahren der Politisierung schwerer wiegen als die Chancen der Entstehung eines gemeinsamen politischen Raums. Stefano Bartolini: Mass Politics in Brussels: How Benign Could It Be?, in: Zeitschrift für Staats- und Europawissenschaften 2/2006, S. 28-56, hier S. 47. 
Die in diesem Beitrag vorgenommene Bestandsaufnahme der europäischen Integration offenbart ein Dilemma: Nur solche EU-Initiativen, die Konflikte hervorrufen, haben Politisierungspotenzial. Sie überspringen die Wahrnehmungsschwelle, lösen öffentliche Debatten und zum Teil Proteste aus. Doch die durch sie bewirkte Politisierung führt nicht ohne Weiteres zu erhöhter Sensibilität gegenüber den Argumenten der Gegenseite, sondern schürt Integrationsskepsis auf Seiten derjenigen, die befürchten, zu den Verlierern einer weiteren Marktintegration zu gehören. Sowohl eine wachsende Distanz zur europäischen Einigung als auch der Ruf nach Protektionismus könnten die Folge sein. Was zu dem Fazit führt: Die europäische Wirtschaftsintegration hat eine Tiefe erreicht, in der weniger im Einzelfall mehr sein könnte. Dies gilt insbesondere in jenen Bereichen, in denen Marktintegration durch richterliche Fortentwicklung europäischen Rechts und damit ohne direkte politische Beteiligung der Mitgliedstaaten und des Europäischen Parlaments vorangetrieben wird. ${ }^{80}$

Im Prinzip liegen für die Kommission alle Instrumente bereit, um weitere Bereiche der öffentlichen Daseinsvorsorge - vom öffentlich-rechtlichen Rundfunk über das Krankenhauswesen bis hin zum öffentlichen Sparkassen- und Bildungswesen - dem europäischen Wettbewerbsrecht zu unterwerfen. Auch der EuGH verfügt über die juristischen Mittel, weitere Unterschiede zwischen den europäischen Produktions- und Verteilungsregimen als Behinderungen der Binnenmarktfreiheiten zu interpretieren und auf diesem Wege beispielsweise auf eine Harmonisierung des Gesellschaftsrechts oder der Arbeitsverfassungen hinzuwirken. So wie sich die jüngere EuGH-Rechtsprechung in den Fällen ,Viking ' und ,Laval` als Signal zur Bereitschaft der Subordination der nationalen Arbeits- und Sozialverfassungen unter die Binnenmarktfreiheiten verstehen lässt, müssen die Urteile zum Gesellschaftsrecht und zu den ,Goldenen Aktien' als Ankündigungen verstanden werden, das gesamte Aktien- und Unternehmensrecht der Mitgliedstaaten nach und nach den Binnenmarktregeln zu unterwerfen. Ähnliches gilt für die sich beschleunigende Rechtsprechung zur Antidiskriminierung. Eine entscheidende Frage ist deshalb, wie sensibel die ,Integration durch Recht" auf die von ihr erzeugten politischen Folgen und deren Rückwirkungen auf den Integrationsprozess selbst reagieren kann. Ob Kommission und EuGH in der Lage sind, mehr Responsivität zu entwickeln als in der jüngeren Vergangenheit - und wie, wenn überhaupt, die Begünstigung solcher Responsivität von außen möglich ist -, erscheint offen. Die Antwort auf diese Frage könnte über den künftigen Erfolg des europäischen Integrationsprojekts entscheiden. Denn weitere Überdehnungen der Grundfreiheiten, des Wettbewerbsrechts und mithin auch der europäischen Grundsätze zur Antidiskriminierung könnten dem erreichten Stand der politischen Integration einen Bärendienst erweisen.

80 Die Schaffung eines europäischen Antidiskriminierungsraums bildet hier keine Ausnahme, wie die lebhaften Proteste gegen die umstrittene, Mangold'-Entscheidung des EuGH zeigen. Vergleiche stellvertretend für viele andere: Lüder Gerken (mit Roman Herzog): Stoppt den EuGH!, in: Frankfurter Allgemeine Zeitung, 08.09.2008. 